\title{
Some Analytics on Bias in DSVARs
}

\author{
Martial Dupaigne \\ University of Western Brittany and TSE (GREMAQ) \\ Patrick Fève* \\ University of Toulouse and TSE (GREMAQ and IDEI) \\ and Banque de France (Research Division) \\ Julien Matheron \\ Banque de France (Research Division)
}

January 12, 2007

\begin{abstract}
This paper examines the ability of Structural Vector Autoregressions (SVARs) to properly uncover the impulse response functions of hours after a technology improvement. Using a simple model in which hours do not react to technology shocks, we determine the main sources of distortions in a SVAR model which includes labor productivity growth and labor input in first difference.
\end{abstract}

Keywords: Structural Vector Autoregressions, Hours Worked, Long-Run Restrictions, Biais

JEL Class.: E24, E32

* Address: GREMAQ-Université de Toulouse I, manufacture des Tabacs, Aile J.J. Laffont, bât. F, 21 allée de Brienne, 31000 Toulouse, France. email: patrick.feve@univ-tlse1.fr. We would like to thank F. Collard, A. Guay, H. Le Bihan, F. Mihoubi, F. Portier and H. Ulhig for discussions, and an anonymous referee for helpful comments. The traditional disclaimer applies. The views expressed herein are those of the authors and not necessarily those of the Banque de France. 


\section{Introduction}

The response of hours to a technology shock is the subject of many controversies in quantitative macroeconomics. Galí (1999) and Francis and Ramey (2004) show that the short-run response of hours to a technology shock is significantly negative in the US economy. They obtain this result using a Structural Vector Autoregression (SVAR) of labor productivity growth and hours in first difference (DSVAR) with long-run restriction (Blanchard and Quah, 1989). However, recent contributions proceeding with simulation experiments have shown that the DSVAR specification can induce large distortions, suggesting that the estimated response from DSVAR can be heavily downward biased (Erceg, Guerrieri and Gust, 2005, Chari, Kehoe and McGrattan, 2005).

This paper makes some analytical progress on this issue. We use a simple model as the Data Generating Process and investigate under which conditions DSVARs may lead to unbiased estimates of the effects of technology improvements. The model is sufficiently simple to clearly locate the main sources of distortions in DSVARs. ${ }^{1}$

The paper is organized as follow. Section 1 expounds the model. Section 2 presents and discusses our results. The last section briefly concludes.

\section{The Model}

We consider a flex price version of the simple model analyzed in Galí (1999). The representative household seeks to maximize

$$
\log \left(C_{t}\right)+\bar{\chi} \exp \left(\chi_{t}\right)\left(1-N_{t}\right), \quad \bar{\chi}>0,
$$

subject to the per period budget constraint $C_{t} \leq W_{t} N_{t}+\Pi_{t}$. The quantity of good consumed in period $t$ is $C_{t}$. The variable $N_{t}$ denotes hours worked, $W_{t}$ is the real wage, and $\Pi_{t}$ represents the profit that the household receives from the firm. The utility function is separable, logarithmic in consumption, and without loss of generality linear in leisure. Finally, $\chi_{t}$ is a random variable that shifts utility every periods. This variable is assumed to follow an $\mathrm{AR}(1)$ process

$$
\chi_{t}=\rho_{\chi} \chi_{t-1}+\sigma_{\chi} \varepsilon_{\chi, t},
$$

where $\varepsilon_{\chi, t}$ is iid with zero mean and unit variance. As noticed by Galí (2005), this shock can be an important source of fluctuations, as it accounts for persistent shifts in

\footnotetext{
${ }^{1}$ Our results only apply to SVARs that identify technology shocks and ignores the widely used monetary VARs, which rely on short-run restrictions.
} 
the marginal rate of substitution between goods and labor. Such shifts capture persistent fluctuations in labor supply following changes in labor market participation and/or changes in the demographic structure. ${ }^{2}$

The representative firm produces a homogenous good with a technology

$$
Y_{t}=Z_{t} N_{t}^{\alpha}
$$

where $\alpha \in(0,1]$. $Z_{t}$ follows a random walk

$$
Z_{t}=Z_{t-1} \exp \left(\sigma_{z} \varepsilon_{z, t}\right)
$$

where $\varepsilon_{z, t}$ is iid with zero mean and unit variance. From the households and firms optimality conditions and market clearing $Y_{t}=C_{t}=Z_{t} N_{t}^{\alpha}$, equilibrium employment $N_{t}$ and labor productivity $X_{t}$ are given by $N_{t}=\alpha \exp \left(-\chi_{t}\right) / \bar{\chi}$ and $X_{t}=Z_{t}(\bar{\chi} / \alpha)^{1-\alpha} \exp \left(\chi_{t}\right)^{1-\alpha}$. Taking logs and ignoring constant terms, we obtain the following log-linear representation of the economy

$$
\begin{aligned}
n_{t} & =-\chi_{t}, \\
\Delta x_{t} & =\sigma_{z} \varepsilon_{z, t}+(1-\alpha) \Delta \chi_{t}, \\
\chi_{t} & =\rho_{\chi} \chi_{t-1}+\sigma_{\chi} \varepsilon_{\chi, t},
\end{aligned}
$$

where $\Delta$ is the first difference operator and lower case letters represent the logarithms of the associated variables. In this economy, hours worked (2) do not react to a technological shock but decrease after a preference shock. Labor productivity (3) jumps permanently after the technological shock. The stationary preference shock (4) has a positive impact effect on labor productivity, but no long-run effect.

\section{Identification from DSVAR model}

Following Galí (1999), Galí and Rabanal (2004), and Francis and Ramey (2004), we consider the following DSVAR(1) model

$$
\mathbf{z}_{t}=\mathbf{A}_{1} \mathbf{z}_{t-1}+\varepsilon_{t}, \quad \mathrm{E}\left\{\varepsilon_{t} \varepsilon_{t}^{\prime}\right\}=\Sigma
$$

with $\mathbf{z}_{t}=\left(\Delta x_{t}, \Delta n_{t}\right)^{\prime}$. In order to get analytical results, we only consider a $\operatorname{VAR}(1)$ model. Despite its simplicity, this assumption allows us to shed light on the main mechanisms at work during the course of identification. Let us define $\mathbf{B}(L)=\left(\mathbf{I}_{2}-\mathbf{A}_{1} L\right)^{-1}$, so that

\footnotetext{
${ }^{2}$ Note that this shock is observationally equivalent to a tax on labor income.
} 
$\mathbf{z}_{t}=\mathbf{B}(L) \varepsilon_{t}$, where $\mathbf{I}_{2}$ is the identity matrix. We assume that the canonical innovations $\varepsilon_{t}$ are linear combinations of the structural shocks $\boldsymbol{\eta}_{t}$, i.e. $\varepsilon_{t}=\mathbf{S} \boldsymbol{\eta}_{t}$, for some nonsingular matrix S. As usual, we impose an orthogonality assumption on the structural shocks which, together with a scale normalization, implies $\mathrm{E} \boldsymbol{\eta}_{t} \boldsymbol{\eta}_{t}^{\prime}=\mathbf{I}_{2}$. This gives us three constraints out of the four needed to completely identify $\mathbf{S}$. To setup the last identifying constraint, let us define $\mathbf{C}(L)=\mathbf{B}(L) \mathbf{S}$. Given the ordering of $\mathbf{z}_{t}$, we simply require that $\mathbf{C}(1)$ be lower triangular, so that only technology shocks can affect the long-run level of labor productivity. This amounts to imposing that $\mathbf{C}(1)$ is the Cholesky factor of $\mathbf{B}(1) \boldsymbol{\Sigma} \mathbf{B}(1)^{\prime}$. Given consistent estimates of $\mathbf{B}(1)$ and $\boldsymbol{\Sigma}$, we easily obtain an estimate for $\mathbf{C}(1)$. Retrieving $\mathbf{S}$ is then a simple task using the formula $\mathbf{S}=\mathbf{B}(1)^{-1} \mathbf{C}$ (1). The impulse response functions are then deduced from the $\operatorname{VMA}(\infty)$ representation $\mathbf{z}_{t}=$ B $(L) \mathbf{B}(1)^{-1} \mathbf{C}(1) \boldsymbol{\eta}_{t}$ with $\boldsymbol{\eta}_{t}=\left(\boldsymbol{\eta}_{1, t}, \boldsymbol{\eta}_{2, t}\right)^{\prime}$, where $\boldsymbol{\eta}_{1, t}$ is the identified technology shock, whereas $\boldsymbol{\eta}_{2, t}$ is the non-technology one.

We use the system (2)-(4) as the Data Generating Process. Given the realization of the equilibrium, we seek to evaluate the quantitative implications of the DSVAR(1) specification when the econometrician uses long-run restrictions on labor productivity in order to uncover the effect of a technology shock on employment. The following proposition (the proof is in appendix) characterizes the impulse response function of hours to a technology shock.

Proposition 1 The impulse response function of hours worked to a permanent shock to labor productivity $\boldsymbol{\eta}_{1, t}$ in a DSVAR(1) model under the model (2)-(4) is given by

$$
\frac{\partial n_{t+k}}{\partial \boldsymbol{\eta}_{1, t}}=-\frac{(1-\alpha) \sigma_{\chi}^{2}}{\left(\sigma_{z}^{2}+\frac{2(1-\alpha)^{2}}{3-\rho_{\chi}} \sigma_{\chi}^{2}\right)^{1 / 2}} \frac{1-\left(\frac{\rho_{\chi}-1}{2}\right)^{k+1}}{1+\frac{1-\rho_{\chi}}{2}} .
$$

We discuss below some implications of Proposition 1.

First, the estimated response of hours deduced from the DSVAR(1) model is downward biased at all horizons. Hence, an applied researcher using a DSVAR would conclude that hours decrease after a technology shock under this model, where hours do not react. ${ }^{3}$ In some other cases such as models with real or nominal rigidities (see Erceg, Guerrieri and Gust, 2005), the DSVAR works well, i.e. recovers the true negative response of hours.

\footnotetext{
${ }^{3}$ In Chari, Kehoe and McGrattan (2005), the DSVAR identifies a decrease in hours under a frictionless RBC model where hours persistently increase after a technology improvement.
} 
As guides to evaluate and develop dynamic general equilibrium models, the discriminating power of the DSVAR approach is therefore low. The results of Proposition 1 are asymptotic and do not hinge on small sample biases. Note that a DSVAR model is obviously misspecified under the model considered here, as it implies an over-differentiation of hours. The correct specification of hours is in level, denoted LSVAR (see Christiano, Eichenbaum and Vigfusson, 2004). In this model without time-varying capital, only one lag in the LSVAR model is required to fully capture the true dynamics of model (2)(4). Hence, the level specification uncovers the true response of hours to a technology shock. This contrasts with Chari, Kehoe and McGrattan (2005)'s results, which show that omitted state variables are a cause of distorsions.

Second, we consider a DSVAR model with only one lag, to get analytical results. With more lags, the response of hours in the DSVAR would be affected, because the first difference of hours introduces a unit root in the moving average of the preference shock. Numerical experiments (not reported) suggest that the bias decreases with more lags, as in Chari, Kehoe and McGrattan (2005). In the LSVAR specification, adding lags is unnecessary since the model (2)-(4) admits a VAR representation with one lag.

Third, when the variance of the non-technology shock tends to zero, the bias decreases. This second result implies that when technology shock are the main source of aggregate fluctuations, the DSVAR(1) model can properly uncover the true responses of hours. This suggests that part of the simulations results discussed above (Chari, Kehoe and McGrattan, 2005) relative to biases in DSVARs are irrelevant in economies where nontechnology shocks play a minor role (Christiano, Eichenbaum and Vigfusson, 2006).

Fourth, when the labor share $\alpha$ tends to one, the response of hours is zero. In this case, productivity growth depends only on the technology shock and the impulse responses of hours are uniformly zero. In fact, when productivity growth is an appropriate measure of total factor productivity growth, the DSVAR specification uncovers the true response of hours after a technology shock. It follows that the specification of hours, i.e. in level versus in difference, is of no substantive importance. This is confirmed by Basu, Fernald and Kimball (2004) who construct a measure of aggregate technology change, controlling for imperfect competition, varying utilization of factors, and aggregation effects. Using this purified measure of total factor productivity in a SVAR with a long-run restriction, they show that the specification of hours (level versus difference) has very little effect on the estimated response. 


\section{Concluding Remarks}

This paper determines some sources of distortions in DSVARs. We show that the DSVAR model leads to downward estimates of the true impulse response functions of hours worked. Our findings emphasize that VAR estimation results are unbiased as long as hours receive the appropriate treatment. Furthermore, researchers should not use labor productivity but rather should use total factor productivity and only trust their results when the treatment of hours does not matter.

\section{References}

Basu, S., Fernald, J. and M. Kimball (2004) "Are Technology Improvements Contractionary?", forthcoming American Economic Review.

Blanchard, O.J. and D. Quah (1989) "The Dynamic Effects of Aggregate Demand and Supply Disturbances", American Economic Review, 79(4), pp. 655-673.

Chari, V., Kehoe, P. and E. McGrattan (2005) "A Critique of Structural VARs Using Real Business Cycle Theory", Federal Reserve Bank of Minneapolis, Research Department Staff Report 364.

Christiano, L., Eichenbaum, M. and R. Vigfusson (2004) "What Happens after a Technology Shock?", NBER Working Paper Number 9819 revised version 2004.

Christiano, L., Eichenbaum, M. and R. Vigfusson (2006) "Assessing Structural VARs", Northwestern University.

Erceg, C., Guerrieri, L. and C. Gust (2005) "Can Long-Run Restrictions Identify Technology Shocks", Journal of the European Economic Association,3(6), pp.1237-1278.

Francis, N. and V. Ramey (2004) "Is the Technology-Driven Real Business Cycle Hypothesis Dead? Shocks and Aggregate Fluctuations Revisited", Journal of Monetary Economics, 52(8),pp. 1379-1399.

Galí, J. (1999) "Technology, Employment and the Business Cycle: Do Technology Shocks Explain Aggregate Fluctuations?", American Economic Review, 89(1), pp. 249-271. 
Galí, J. (2005) "Trends in Hours Worked and the Role of Technology in the Business Cycle Theory: Theory and International Evidence", Federal Reserve Bank of St. Louis Review, July/August 2005, 87(4), pp. 459-86.

Galí, J., and P. Rabanal (2004) "Technology Shocks and Aggregate Fluctuations: How Well does the RBC Model Fit Postwar U.S. Data?", NBER Macroeconomics Annual, Gertler and Rogoff, eds., pp.225-288. 


\section{Appendix}

We consider the estimation of a $\operatorname{VAR}(1)$ model

$$
\left(\begin{array}{c}
\Delta x_{t} \\
\Delta n_{t}
\end{array}\right)=\mathbf{A}_{1}\left(\begin{array}{c}
\Delta x_{t-1} \\
\Delta n_{t-1}
\end{array}\right)+\left(\begin{array}{c}
\varepsilon_{1, t} \\
\varepsilon_{2, t}
\end{array}\right), \quad \mathbf{A}_{1}=\left(\begin{array}{ll}
\boldsymbol{a}_{11} & \boldsymbol{a}_{12} \\
\boldsymbol{a}_{21} & \boldsymbol{a}_{22}
\end{array}\right),
$$

with data generated by the structural model (2)-(4). The plim of the OLS estimators in the first and second equations are

$$
\begin{aligned}
& \left(\begin{array}{l}
a_{11} \\
a_{12}
\end{array}\right)=\left(\begin{array}{cc}
V\left(\Delta x_{t}\right) & \operatorname{Cov}\left(\Delta x_{t}, \Delta n_{t}\right) \\
\operatorname{Cov}\left(\Delta x_{t}, \Delta n_{t}\right) & V\left(\Delta n_{t}\right)
\end{array}\right)^{-1}\left(\begin{array}{c}
\operatorname{Cov}\left(\Delta x_{t}, \Delta x_{t-1}\right) \\
\operatorname{Cov}\left(\Delta x_{t}, \Delta n_{t-1}\right)
\end{array}\right), \\
& \left(\begin{array}{l}
a_{21} \\
a_{22}
\end{array}\right)=\left(\begin{array}{cc}
V\left(\Delta x_{t}\right) & \operatorname{Cov}\left(\Delta x_{t}, \Delta n_{t}\right) \\
\operatorname{Cov}\left(\Delta x_{t}, \Delta n_{t}\right) & V\left(\Delta n_{t}\right)
\end{array}\right)^{-1}\left(\begin{array}{l}
\operatorname{Cov}\left(\Delta n_{t}, \Delta x_{t-1}\right) \\
\operatorname{Cov}\left(\Delta n_{t}, \Delta n_{t-1}\right)
\end{array}\right) .
\end{aligned}
$$

The variances and covariances which enter the $\mathbf{A}_{1}$ matrix are given by $V\left(\Delta x_{t}\right)=\sigma_{z}^{2}+2 \sigma_{\chi}^{2}(1-\alpha)^{2} /\left(1+\rho_{\chi}\right)$, $V\left(\Delta n_{t}\right)=2 \sigma_{\chi}^{2} /\left(1+\rho_{\chi}\right), \operatorname{Cov}\left(\Delta x_{t}, \Delta n_{t}\right)=-2(1-\alpha) \sigma_{\chi}^{2} /\left(1+\rho_{\chi}\right), \operatorname{Cov}\left(\Delta x_{t}, \Delta x_{t-1}\right)=-\left(1-\rho_{\chi}\right)(1-$ $\alpha)^{2} \sigma_{\chi}^{2} /\left(1+\rho_{\chi}\right), \operatorname{Cov}\left(\Delta x_{t}, \Delta n_{t-1}\right)=\left(1-\rho_{\chi}\right)(1-\alpha) \sigma_{\chi}^{2} /\left(1+\rho_{\chi}\right), \operatorname{Cov}\left(\Delta n_{t}, \Delta x_{t-1}\right)=\left(1-\rho_{\chi}\right)(1-\alpha) \sigma_{\chi}^{2} /(1+$ $\left.\rho_{\chi}\right)$ and $\operatorname{Cov}\left(\Delta n_{t}, \Delta n_{t-1}\right)=-\left(1-\rho_{\chi}\right) \sigma_{\chi}^{2} /\left(1+\rho_{\chi}\right)$. The plim of the OLS estimator of $\mathbf{A}_{1}$ is then

$$
\mathbf{A}_{1}=\left(\begin{array}{cc}
0 & \frac{\left(1-\rho_{\chi}\right)(1-\alpha)}{2} \\
0 & -\frac{1-\rho_{\chi}}{2}
\end{array}\right) .
$$

The covariance matrix of the canonical residuals $\left\{\varepsilon_{1, t}, \varepsilon_{2, t}\right\}$ is

$$
\boldsymbol{\Sigma}=\left(\begin{array}{cc}
\sigma_{z}^{2}+\frac{\left(3-\rho_{\chi}\right)\left(1-\alpha^{2}\right)}{2} \sigma_{\chi}^{2} & -\frac{\left(3-\rho_{\chi}\right)(1-\alpha)}{2} \sigma_{\chi}^{2} \\
-\frac{\left(3-\rho_{\chi}\right)(1-\alpha)}{2} \sigma_{\chi}^{2} & \frac{3-\rho_{\chi}}{2} \sigma_{\chi}^{2}
\end{array}\right)
$$

Using $A_{1}$ and $\Sigma$, we compute the long-run covariance matrix $\Omega=\left[\left(\mathbf{I}_{2}-\mathbf{A}_{1}\right)^{-1}\right] \mathbf{\Sigma}\left[\left(\mathbf{I}_{2}-\mathbf{A}_{1}\right)^{-1}\right]^{\prime}$

$$
\begin{aligned}
\Omega & =\left(\begin{array}{cc}
1 & \frac{\left(1-\rho_{\chi}\right)(1-\alpha)}{3-\rho_{\chi}} \\
0 & \frac{2}{3-\rho_{\chi}}
\end{array}\right)\left(\begin{array}{cc}
\sigma_{z}^{2}+\frac{\left(3-\rho_{\chi}\right)\left(1-\alpha^{2}\right)}{2} \sigma_{\chi}^{2} & -\frac{\left(3-\rho_{\chi}\right)(1-\alpha)}{2} \sigma_{\chi}^{2} \\
-\frac{\left(3-\rho_{\chi}\right)(1-\alpha)}{2} \sigma_{\chi}^{2} & \frac{3-\rho_{\chi}}{2} \sigma_{\chi}^{2}
\end{array}\right)\left(\begin{array}{cc}
1 & 0 \\
\frac{\left(1-\rho_{\chi}\right)(1-\alpha)}{3-\rho_{\chi}} & \frac{2}{3-\rho_{\chi}}
\end{array}\right) \\
& =\left(\begin{array}{cc}
\sigma_{z}^{2}+\frac{2(1-\alpha)^{2}}{3-\rho_{\chi}} \sigma_{\chi}^{2} & -\frac{2(1-\alpha)}{3-\rho_{\chi}} \sigma_{\chi}^{2} \\
-\frac{2(1-\alpha)}{3-\rho_{\chi}} \sigma_{\chi}^{2} & \frac{2}{3-\rho_{\chi}} \sigma_{\chi}^{2}
\end{array}\right) .
\end{aligned}
$$

The matrix $\boldsymbol{C}(1)$ is the Choleski decomposition of the long-run covariance matrix

$$
\mathbf{C}(1)=\left(\begin{array}{cc}
\left(\sigma_{z}^{2}+\frac{2(1-\alpha)^{2}}{3-\rho_{\chi}} \sigma_{\chi}^{2}\right)^{1 / 2} & 0 \\
-\frac{2(1-\alpha) \sigma_{\chi}^{2}}{\left(3-\rho_{\chi}\right)\left(\sigma_{z}^{2}+\frac{2(1-\alpha)^{2}}{3-\rho_{\chi}} \sigma_{\chi}^{2}\right)^{1 / 2}} & \left(\frac{2 \sigma_{z}^{2} \sigma_{\chi}^{2}}{\left(3-\rho_{\chi}\right)\left(\sigma_{z}^{2}+\frac{2(1-\alpha)^{2}}{3-\rho_{\chi}} \sigma_{\chi}^{2}\right)}\right)^{1 / 2}
\end{array}\right) .
$$

The impulse response functions of labor productivity and hours are then deduced from $\mathbf{C}(L)=\left(\mathbf{I}_{2}-\right.$ $\left.\mathbf{A}_{1} L\right)^{-1}\left(\mathbf{I}_{2}-\mathbf{A}_{1}\right) \mathbf{C}(1)$. The response of hours worked at horizon $k$ is given by

$$
-\frac{(1-\alpha) \sigma_{\chi}^{2}}{\left(\sigma_{z}^{2}+\frac{2(1-\alpha)^{2}}{3-\rho_{\chi}} \sigma_{\chi}^{2}\right)^{1 / 2}} \sum_{j=0}^{k}\left(-\left(\frac{1-\rho_{\chi}}{2}\right)\right)^{j} .
$$

Since $\left|\left(1-\rho_{\chi}\right) / 2\right|<1$, we obtain

$$
\sum_{j=0}^{k}\left(-\left(\frac{1-\rho_{\chi}}{2}\right)\right)^{j}=\frac{1-\left(\frac{\rho_{\chi}-1}{2}\right)^{k+1}}{1+\frac{1-\rho_{\chi}}{2}}>0
$$

The response of hours is negative for any horizon. This completes the proof. 